\title{
Primitive pleural leiomyosarcoma: Case report
}

\author{
Layla Belliraj*1, Sani Zakari Rabiou ${ }^{1}$, Ibrahim Issoufou Alzouma ${ }^{1}$, Jamal Ghalimi ${ }^{1}$, Fatima Zohra Ammor ${ }^{1}$, Marouane \\ Lakranbi $^{1}$, Yassine Ouadnouni ${ }^{1,2}$, Mohamed Smahi, ${ }^{1,2}$ \\ ${ }^{1}$ Department of Thoracic Surgery, Universitary Hospital Hassan II Fes, Morocco \\ ${ }^{2}$ Faculty of Medicine and Pharmacy, University Sidi Mohamed Ben Abdellah, Fes, Morocco
}

Received: September 28, 2015

DOI: $10.5430 /$ jst.v6n1p62
Accepted: December 1, $2015 \quad$ Online Published: January 19, 2016

URL: http://dx.doi.org/10.5430/jst.v6n1p62

\begin{abstract}
Leiomyosarcoma is a malignant tumor of smooth muscle, primitive pleural localization is extremely rare, to our knowledge only ten cases have been reported in the literature. The prognosis is usually grim, the survival of patients with extensive disease is about 12 months. Our patient has survived 42 months which is rarely reported in the literature.
\end{abstract}

Key Words: Leiomyosarcoma, Pleural, Surgery

\section{INTRODUCTION}

Primary sarcomas of the thorax are rare. They occur in the lung, mediastinum, pleura, and chest wall. Angiosarcoma, leiomyosarcoma, rhabdomyosarcoma, and mesothelioma are the most common primary intrathoracic sarcomas.

Primitive pleural leiomyosarcoma is extremely rare, there are few reports of individual cases, but no large series from a single institution has been published.

Given the scarcity of primary pleural leiomyosarcoma and its clinical and histological similarities with other pleural malignancies, including sarcomatoid mesothelioma, diagnosis is difficult. ${ }^{[1,2]}$

\section{CASE}

A 54-year-old North African man, smoker and chronic alcoholic, already had surgery for histiocytoma of the left knee 9 years ago, who consulted for hemoptysis associated with chest pain for the previous three months, and in whom clinical examination has shown pulmonary condensation syndrome of the right base of the thorax.
A chest radiograph showed a right opacity of the base of the thorax (see Figure 1). Chest computed tomography (CT) showed a right pleural tumor infiltrating the right lower lobe and repressing the inferior vena cava (IVC), the liver and the diaphragm (see Figure 2).

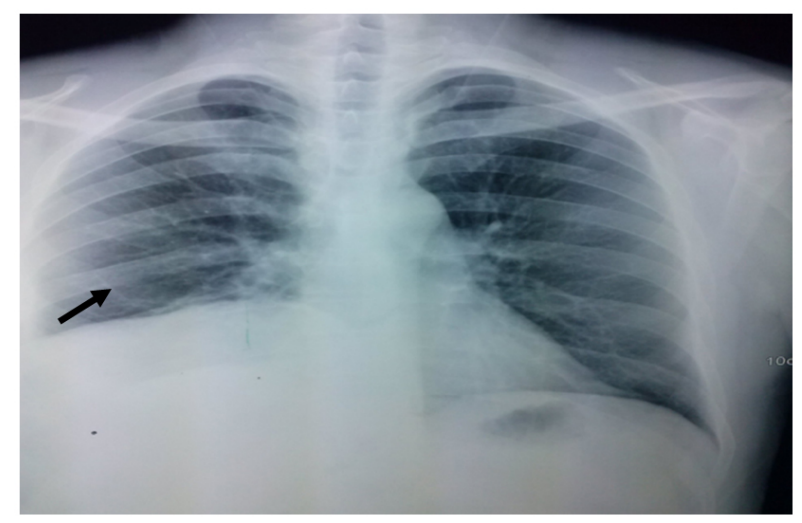

Figure 1. Chest radiograph showing a right basal opacity which merges with the walls and diaphragm

Bronchoscopy showed incomplete stenosis of the right para-

\footnotetext{
*Correspondence: Layla Belliraj; Email: lbelliraj@yahoo.fr; Address: Department of Thoracic Surgery, Universitary Hospital Hassan II Fes, Morocco.
} 
cardiac bronchus by extrinsic compression and complete stenosis of the posterior basal segmental.

The patient underwent a right lower lobectomy extended to the diaphragm with phrenoplasty. Pathological examination of the surgical specimen has shown a malignant tumor proliferation, sometimes arranged in long beams crossed at right angles sometimes in diffuse sheets, with fusiform tumor cells with enlarged, irregular and hyperchromatic nuclei and clear or eosinophilic cytoplasm.

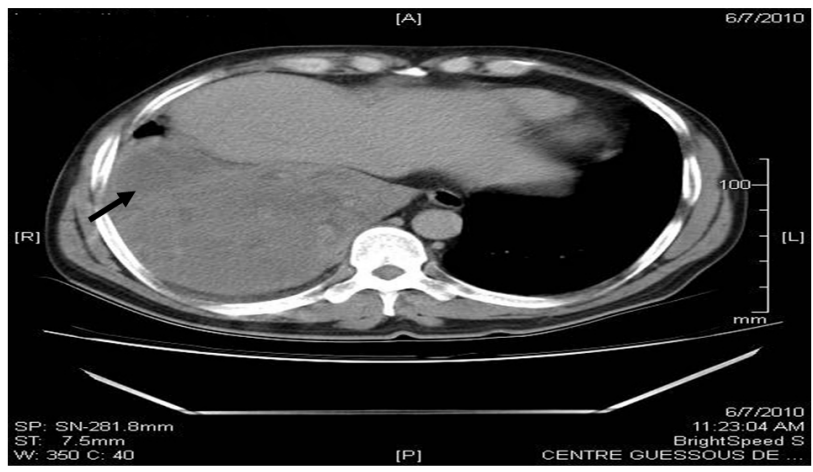

Figure 2. CT image showing a right basal mass with liquefied tissular density that enhances after injection

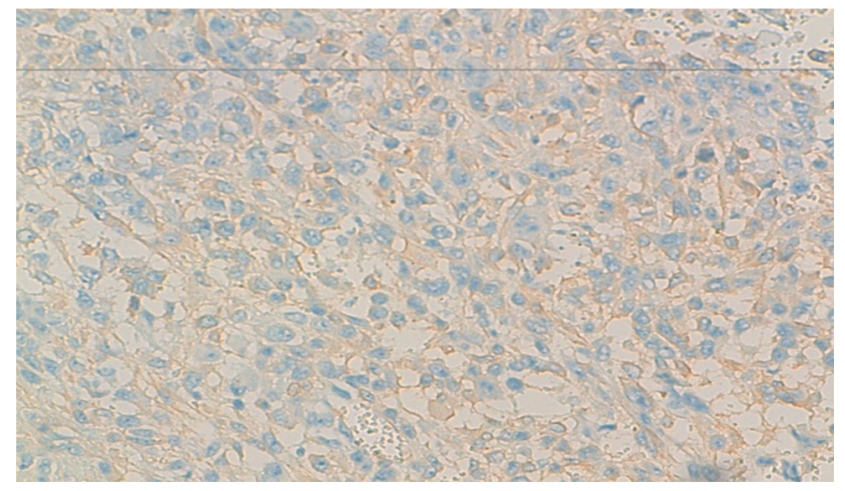

Figure 3. Positive immunostaining (cytoplasmic) of tumor cells by antibodies AML (smooth muscle actin): Sarcomatous spindle cell proliferation with positive immunogold antimuscle smooth antibodies

Immunohistochemistry (IHC) staining was performed. The tumor cells were positive for AML(anti smooth muscle actin), Hcal desmone, actin, S100 protein, Epithelial membrane antigen (EMA) and Melan A was positive, which was in favor of pleural leiomyosarcoma grade II of the FNCLCC (see Figures 3 and 4). After discussion in a multidisciplinary meeting, adjuvant chemotherapy was started with IA protocole: Ifosfamide + Adriamycin, the patient received four chemotherapy cycles. The clinical and radiological control of 3, 6 and 12 months didn't reveal any tumoral progression.

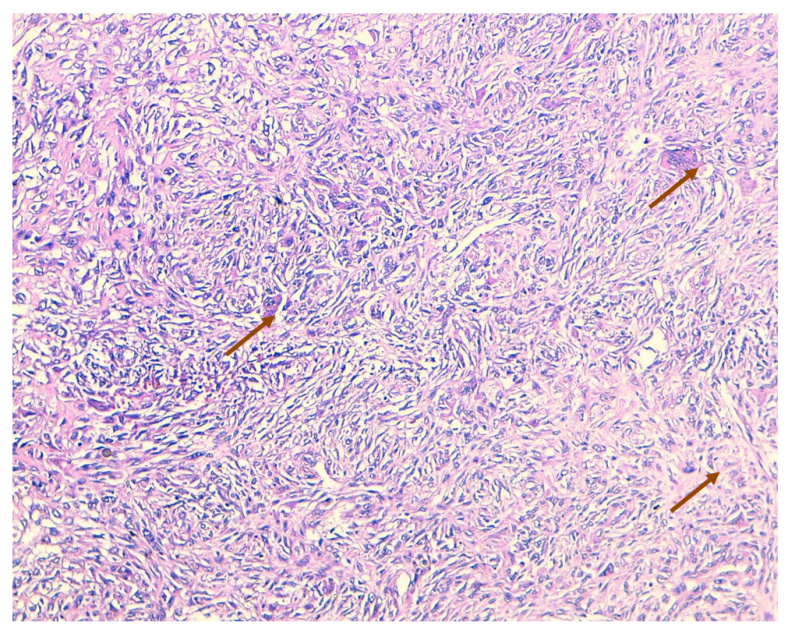

Figure 4. HES $\times 10$ Proliferation fusocellulaire arranged diffuse layers of intersecting beams: The cell density is high, The cells are frankly atypical with an elongate core cut at right angles "cigarette butt", a dense chromatin and eosinophilic cytoplasm. Note the presence of numerous multinucleate cells (arrow). Several mitotic figures are also noted (arrow).

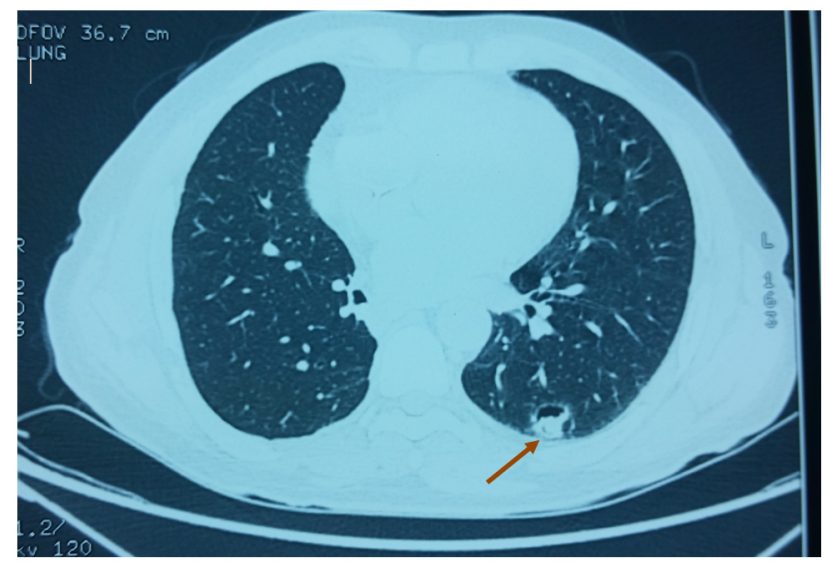

Figure 5. CT image: a caviarty lesion which became irregular and nodular (arrow)

After 18 months, thoraco-abdomino-pelvic and brain CT control showed an increase in the volume of the left lower lobar lesion, of which the wall became irregular and the appearance of a nodular lesion in this cavity (see Figure 5). The PET scan confirmed the hyper-metabolic nature of the lesion. In multidisciplinary meeting the decision was to perform surgical exploration. The patient benefited a left lower lobectomy (after trans-parietal biopsy in favor of a lung metastasis of the leiomyosarcoma).

Even so, a CT scan of the whole body was performed at 36 months showed locoregional recurrence with medullary involvement. Additional thoracic magnetic resonance imaging 
(MRI) showed an increase in para-vertebral lesion with epidural infiltration, and therefore the patient received a secondline chemotherapy with Carboplatin - Paclitaxel (4 cycles), than Docetaxel- Gemcitabin (5 cycles), combined with short course decompressive radiotherapy.

There was no clinical improvement, therefore a surgical decompression has been decided, but the patient died on day +12 after postoperative septic shock following a bacterial meningitis.

\section{Discussion}

Leiomyosarcomas are malignant tumors of smooth muscle that occur most often in uterus, gastrointestinal tract, or soft tissue. Pleural origin is still very rare, until now, no large series has documented the exact number of pleural leiomyosarcoma in the world.

Leiomyosarcomas preferentially occur in adulthood, no risk factors or specific exposure is highlighted. ${ }^{[1]}$

Clinically it is manifested by respiratory symptoms that remains not specific and varies depending on the tumor location and development, ${ }^{[1,3]}$ in our case it resulted in low abundance hemoptysis and chest pain.

In CT scan, tumor appears as a heterogeneous tissue mass, enhanced inhomogeneously after contrast injection, as in our case. In MRI, in T1 sequence, the tumor has intermediate signal which is getting stronger in T2. ${ }^{[1]}$

Only a biopsy of the tumor process can confirm the diagnosis, however, it must be done either by thoracoscopy or thoracotomy. The primitive pleural origin is confirmed after having eliminated all other locations by a thoraco-abdominal-pelvic CT. ${ }^{[2]}$

In pathology, cells are arranged in long beams intersecting at right angles, with abundant eosinophilic cytoplasm and often fibrillar, optionally containing a vacuole notching the kernel. The nucleus is central, rounded in "cigar". The cores may be irregular, polymorphic multilobed or monstruous. Rarely available palisade cores may impose for schwann tumor.

The mitotic index is the best criterion for malignancy of these

\section{REFERENCES}

[1] Ouadnouni Y, Bouchikh M, Jahid A, et al. Leiomyosarcome pleural primitif (A PROPOS D’UN CAS) ouvin médicale. 2010; 129(6): 241-3.

[2] Rais G, Raissouni S, Mouzount H, et al. Primary pleural leiomyosarcoma with rapid progression and fatal outcome: a case report. Journal of Medical Case Reports. 2012; 6: 101. tumors.

There are forms with areas of pseudo-epithelial cell shapes with anaplastic areas, polymorphic or inflammatory cells, which may resemble a malignant fibrous histiocytoma.

Desmin is the most widely used marker in immunohistochemistry, about $50 \%$ of soft tissue leiomyosarcomas are positive with anti-desmin antibody. The overall muscle actin (HHF35) and especially smooth muscle actin were more often positive but less specific. A new marker, the h-caldesmon seems endowed with good specificity. It is expressed by $85 \%$ of leiomyosarcoma. Positivity for cytokeratin, EMA and $\mathrm{S} 100$ protein is also reported in some cases.

Cytogenetic study may be useful next to IHC for diagnostic confirmation.

Surgery remains the treatment of choice for localized tumor or local extension. Radiotherapy may be propose preoperatively or postoperatively when locoregional recurrences, and although sarcomas are known as the most chemoresistant tumors, a sensitivity of $20 \%$ to doxorubicin, ifosfamide, and trabectedin is described in leiomyosarcomas.

The prognosis is usually grim essentially depends on the histologic grade, the presence of metastasis, and the possibilities of extended surgical resections. The survival of patients with extensive disease is about 12 months. ${ }^{[4]}$ Our patient has survived 42 months which is rarely reported in the literature.

\section{Conclusion}

Primitive pleural leiomyosarcoma remains a very rare tumor. Only pathology can confirm the diagnosis, after eliminating a well distinct localization that can be responsible for pleural disease. Surgery is curative treatment, but the prognosis is generally pejorative depending on the possibilities of complete surgical resection.

\section{ACKNOWLEDGEMENTS}

The authors thank the family's patient. We also thank the radiology department and pathology department.

\section{CONFLicts OF InTEREST Disclosure}

The authors declare that they have no competing interests.
[3] Gladish GW, Sabloff BM, Mundenet RF, et al. Primary Thoracic Sarcomas. RSNA. 2002.

[4] Yajnik S, Rosenzweig KE, Mychalczak B, et al. Hemithoracic radiation after extrapleural pneumonectomy for malignant pleural mesothelioma. J Radiat Oncol Biol Phys. 2003; 56: 1319-26. http://dx.doi.org/10.1016/S0360-3016(03)00287-6 